\title{
LA DEUDA EXTERNA PUBLICA PERUANA 1970-2003
}

\section{PERUVIAN PUBLIC FOREIGN DEBT 1970-2003}

\author{
José Rospigliosi Ferro ${ }^{1}$
}

\section{RESUMEN}

Entre el año 1970 y el 2003 la Deuda Externa Pública Peruana de mediano y largo plazo, ha recepcionado de los países extranjeros un monto total de $\$ 32656$ millones de dólares, habiéndose amortizado $\$ 31513$ millones de dólares y pagando como intereses el monto de \$26549 millones de dólares que significa un servicio de la deuda externa por \$58 062 millones de dólares. Esto ha significado una descapitalización del país por este rubro en \$25 406 millones de dólares en este periodo, que hace un promedio anual de 770 millones que muestran que salen más recursos que los que ingresan al país, lo que significa una falacia de la denominada "ayuda externa". El periodo de mayor descapitalización ha sido entre 1986 y 1990.

Palabras clave: Economía peruana, deuda externa, Perú, ingreso nacional, remuneraciones, utilidades.

\begin{abstract}
Between 1970 and 2003 the medium and large term Peruvian Public Foreign Debt had receiving of foreign countries an total amount of \$ 32656 million, having amortized \$ 31513 million and having paid as interests a amount of \$26549 million, that is meaning a foreign extern service of $\$ 58062$. This is a minus capitalization of Perú for $\$ 25406$ millions in this period, that made an annual average of 770 millions that meaning go out more recourses than be income in the country, and this is a falseness of called "extern aid". The time of greater minus capitalization was between 1986 y 1990.
\end{abstract}

Key words: Peruvian economy, foreign debt, Perú, national revenue, remuneration, utilities

\section{INTRODUCCIÓN}

El trabajo que se presenta a continuación es un ensayo de aproximación, a partir de las estadísticas oficiales, del balance sobre el comportamiento del endeudamiento externo de mediano y largo plazo en el Perú en los últimos 34 años.

La importancia del tema radica en que la los excedentes generados en la economía peruana, en los últimos 30 años, se han orientado al pago de la deuda externa, a través de distintos mecanismos económicos, de los cuales el principal es la tributación nacional.

La hipótesis que dirigió este trabajo fue la de que la deuda externa es el principal mecanismo de dependencia en los últimos 30 años, que genera excedentes nacionales orientados al extranjero y su magnitud impide la orientación de los recursos al desarrollo nacional, así como viene desestructurando la economía y sociedad peruana. Sólo se ha avanzado en el dimensionamiento de los recursos que el país entrega al exterior a costa de su propio desarrollo interno.

\section{METODOLOGIA}

El principal método ha sido el del análisis, descriptivo e inductivo.
Se trabajó la información sobre Deuda Externa a partir de las fuentes oficiales de información, tales como la del Instituto Nacional de Estadística e Información (INEI, 1 a 4), y el Banco Central de Reserva $(B C R)(5,6,7,8)$, que son las entidades que oficialmente manejan y consolidan la información. El INEI se encarga de divulgar la información suministrada por el BCR. La deuda privada no ha sido relevante en los montos de este periodo por lo que se optó por dejarla de lado.

La información sobre la deuda externa pública de mediano y largo plazo, ha seleccionado las siguientes variables:

Desembolso, que comprende los montos de dinero ingresado al país de manera efectiva por préstamos hechos desde el extranjero

Amortización, que comprende los pagos efectuados por el país al extranjero, respecto a los montos desembolsados

Interés, que comprende los pagos efectuados, adicional al monto prestado, como compensación por haber hecho uso del dinero prestado y efectivamente desembolsado.

Se seleccionó el periodo quinquenal, de modo que permita determinar el comportamiento de la deuda asociado a los distintos gobiernos. La informa- ción para los años de cambios de gobierno que se hacen los 28 de julio, para cada quinquenio, cubre una parte del gobierno saliente y del entrante. Para este trabajo se ha acumulado el año que atraviesa dos gobiernos, al gobierno saliente, en la medida que los Presupuestos están determinados por dicho gobierno y son pocos los ajustes que se realizan.

En el intento de ver la relación entre deuda externa e ingreso nacional, se trabajó, adicionalmente, información estadística de distintas fuentes sociales de la Universidad Católica $(9,10)$ y Cuanto $(11,12)$ así como del INEI y del BCR respecto al Ingreso Nacional. Esta información está discontinuada desde 1991, pero fue posible trabajarla desde la información que proporciona el INEI relativo a Valor agregado. De modo que en el tema del Ingreso Nacional, los avances que se han utilizado aquí son referenciales y se han presentado como marco contextual, en el que se ubica la deuda externa.

\section{RESULTADOS}

\section{ANTECEDENTES Y CONTEXTO HISTÓRICO}

En los años previos a la denominada Segunda Guerra Mundial de los años 1939 - 1945, el sistema mundial

${ }^{1}$ CECASUR Centro Científico Cultural de Acción Social Ulises Reátegui 
había entrado en una de sus recurrentes crisis cíclicas de manera más profunda y larga. Keynes planteó un cambio en la concepción liberal de la teoría económica capitalista postulando la necesidad de intervención del Estado en la economía de mercado. En los países dependientes o poco desarrollados, la intervención del Estado adquirió una particularidad: se adquirían y administraban maquinarias obsoletas de los capitales transnacionales, revalorizando el capital cero de dichas empresas.

\section{a. El capital financiero}

En los años 70 se impulsó una nueva estrategia económica mediante el sistema de créditos por todo el mundo, que adquirió especial dinamismo a partir de la crisis del petróleo en 197374. Los créditos crecieron significativamente sin ningún control jurídico nacional o internacional generando un nuevo fenómeno dentro del proceso de terciarización de la sociedad y la economía: a este fenómeno se le conoce como LA BURBUJA FINANCIE$R A$.

La deuda mundial de los países latinoamericanos creció a 100000 millones de dólares en 1975 y hoy se encuentra por los 800000 millones, creciendo en la década del 70 y 80 por encima de la tasa productiva.
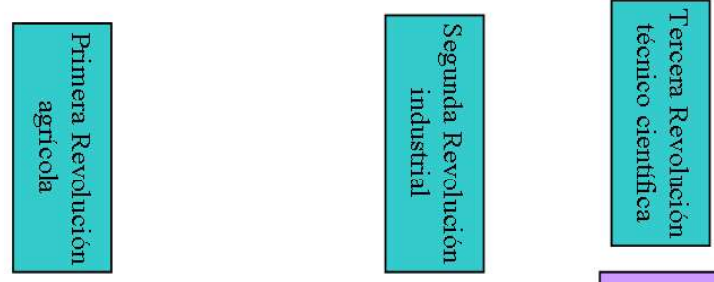

Sociedad post industrial

(conocimiento)

\section{GLOBALIZACION}

Comercio, Finanzas etc Terciarización

\begin{tabular}{|c|c|c|c|c|}
\hline \multirow[t]{4}{*}{10.000 a.c. } & 1780 & 1950 & \multicolumn{2}{|c|}{1970} \\
\hline & manufactura & strial & \multicolumn{2}{|c|}{ servicios } \\
\hline & capitalismo & \multicolumn{3}{|c|}{ IMPERIALISMO } \\
\hline & liberal & $\begin{array}{r}\text { Imperialismo } \\
\text { materias prima }\end{array}$ & $\begin{array}{l}\text { Imperialismo } \\
\text { industrial }\end{array}$ & $\begin{array}{c}\text { Imperialismo Financiero } \\
\text { (deuda externa) }\end{array}$ \\
\hline
\end{tabular}

El capital financiero internacional pasa de un predominante capital exportador productivo (materias primas, industrias sustitutivas para el caso latinoamericano) a un predominio de su modalidad financiera pura (conocida como deuda externa). entregó como pagos por su deuda externa 1 millón de millones 528 mil millones de dólares que duplica el monto de la deuda actual y representó un promedio de pagos anuales por 118 mil millones de dólares. Es decir, cada 6,3 años pagamos la deuda, pero esta carga perversa continúa allí

\begin{tabular}{|c|c|c|c|c|}
\hline 1990 & 1999 & 2000 & $\%$ & $\begin{array}{c}\% \\
\text { PNB }\end{array}$ \\
\hline 1460000 & 2563600 & 2527500 & 100,0 & 37,4 \\
\hline 164100 & 389300 & 376700 & 8,0 & 5,6 \\
\hline 70400 & 122700 & 133600 & 2,9 & 2,0 \\
\hline-10600 & -137300 & -101600 & $-2,2$ & $-1,5$ \\
\hline 4726300 & 6333700 & 6756100 & 144,2 & 100,0 \\
\hline 908200 & 1818700 & 2211200 & 47,2 & 32,7 \\
\hline 475400 & 813400 & 809100 & 32,0 & 38,5 \\
\hline 45600 & 162300 & 167300 & 3,6 & 8,0 \\
\hline 22800 & 50600 & 55500 & 1,2 & 2,6 \\
\hline-2300 & -39300 & -46900 & $-1,0$ & $-2,2$ \\
\hline 1067100 & 1947700 & 2102900 & 44,9 & 100,0 \\
\hline 186800 & 390200 & 468700 & 10,0 & 22,3 \\
\hline
\end{tabular}

inmutable e inextinguible. ¡¡Es una Deuda Eterna!!"

En el caso latinoamericano la deuda creció en $3000 \%$ desde 1970 al $2000,214 \%$ frente a la de 1980 y $70 \%$ respecto a la de 1990 , alcanzando casi el $40 \%$ del PNB en el año 2000 (13).

\section{CONTEXTO EN EL PERU}

Desde 1935, el Estado empieza a intervenir en la economía. Durante el primer gobierno de Belaúnde (196368) los sueldos empiezan un deterioro paulatino, revertido en los primeros años del Gobierno del General Velasco, pero desde 1974 empieza un deterioro irreversible a través de los mecanismos inflacionarios, disminuyendo a cerca de un $10 \%$ de su valor de los años 70 y alcanzando una proporción del $15 \%$ del Ingreso nacional, frente al $30 \%$ en los años 70 y el $50 \%$ a mediados del siglo XX. En contraposición las ganancias se incrementan desde un $19 \%$ a un $48 \%$ en el mismo periodo.

La Fuerza Armada que comandó Velasco Alvarado introduce una serie de cambios en las bases políticas y desarrolla un proyecto centrado en el Estado, contado con el apoyo de sectores del capitalismo financiero internacional que llevan a que la deuda externa pública, entre 1968 y 1975 se elevara desde 623 millones de dólares en 1967 a 6000 millones de dólares en 1980 y desde 1973 estos superávit en la Balanza de Pagos cambiaran en la carga de la deuda externa; esto es, la amortización y los intereses se vuelven altos, lo que llevó 
GRAFICO No 1

INGRESO NACIONAL EN EL PERU 1960-2000 (\%)

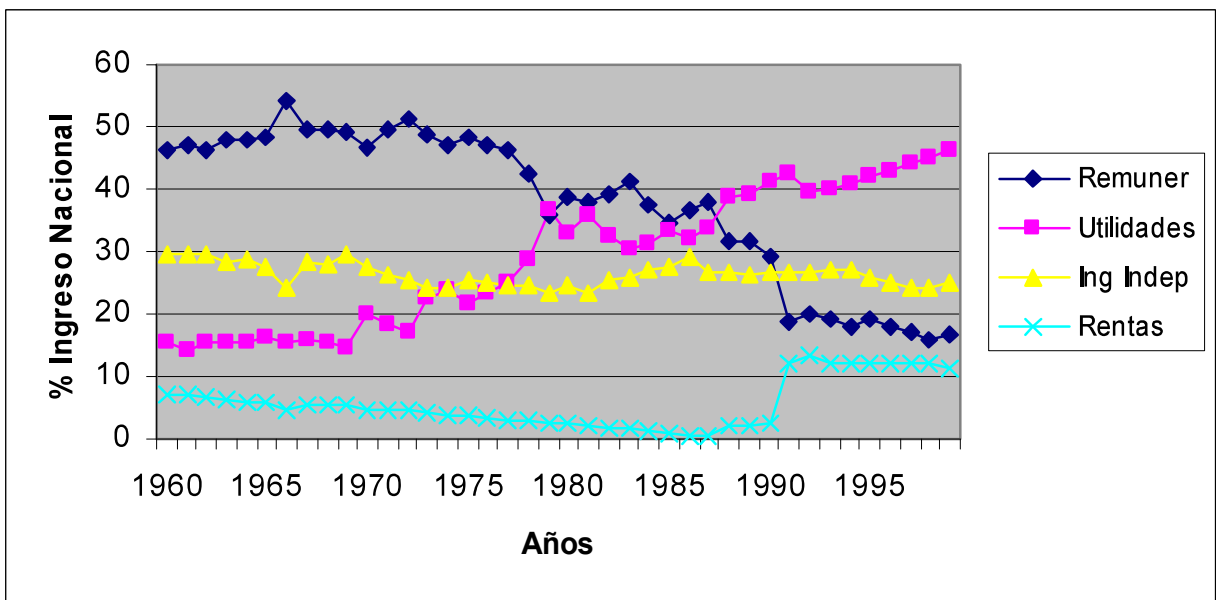

Fuente: 1960 a 1980: I. Yepez delCastillo, J.Bernedo Alvarado. La sindicalización en el Peru (9). 1981 a 1992: Cuanto S.A. Perú en Números 1991 y 1993 (11-12).

a crisis cíclicas en el país. Es en esta etapa donde se instalan las bases de la actual dinámica nacional.

A continuación se presenta el Cuadro $\mathrm{N}^{\circ} 1$, en el cual se ha agrupado por

\section{CUADRO No 1}

DEUDA EXTERNA PÚBLICA PERUANA DE MEDIANO Y LARGO PLAZO

\begin{tabular}{|c|c|c|c|c|c|c|c|}
\hline \multirow[b]{2}{*}{ Período } & \multirow[b]{2}{*}{$\begin{array}{c}\text { Desembol- } \\
\text { so }\end{array}$} & \multirow[b]{2}{*}{$\begin{array}{c}\text { Amortiza- } \\
\text { ción }\end{array}$} & \multirow[b]{2}{*}{ Intereses } & \multirow[b]{2}{*}{$\begin{array}{l}\text { Total } \\
\text { Pago }\end{array}$} & \multirow[b]{2}{*}{$\begin{array}{l}\text { Desem- } \\
\text { Pagos }\end{array}$} & \multicolumn{2}{|c|}{ PROMEDIO ANUAL } \\
\hline & & & & & & $\begin{array}{c}\text { Total } \\
\text { Pagado }\end{array}$ & $\begin{array}{c}\text { Desem- } \\
\text { Pago }\end{array}$ \\
\hline $70-75$ & 3443 & 1415 & 547 & 1962 & 1481 & 327 & 247 \\
\hline $76-80$ & 5004 & 3526 & 1568 & 5095 & -91 & 1019 & -18 \\
\hline $81-85$ & 6803 & 6363 & 3354 & 9717 & -2914 & 1943 & -583 \\
\hline $86-90$ & 2050 & 7049 & 5191 & 12240 & -10190 & 2448 & -2038 \\
\hline $91-95$ & 4102 & 4622 & 7190 & 11812 & -7710 & 2362 & -1542 \\
\hline $96-00$ & 5750 & 4561 & 5530 & 10091 & -4341 & 2018 & -868 \\
\hline $01-03$ & 5505 & 3976 & 3169 & 7145 & -1640 & 2382 & -547 \\
\hline TOTAL & 32656 & 31513 & 26549 & 58062 & -25406 & 1708 & -770 \\
\hline Prom. Anual & 960 & 927 & 781 & 1,708 & -770 & & \\
\hline
\end{tabular}

Fuente.- Banco Central de Reserva. Memorias Anuales varios años y Resumen Mensual (5 a 8) el $0 \%$ de interés), los intereses a pagar sumarian alrededor de 15000 millones de dólares, menor a los 26 mil millones de dólares pagados a la fecha, y con una deuda neta real que debería estar en 4 mil millones de dólares y no en los 22000 millones de dólares, explicable por los procesos de capitalización de intereses que han llevado al incremento de la deuda sin justificación.

El gobierno del APRA con Alan García (1985-90) aplicó políticas keynesianas que no respondieron en la economía. Los capitalistas financieros no le desembolsaron capitales en ese periodo y obligaron a pagar un monto mayor al ofrecido en su campaña electoral y su primer discurso el 28 de julio 1985. El cuadro muestra que fue en este periodo en el que más se amortizó la deuda externa (7 049 millones de dólares) y donde menos recursos se obtuvieron del capital financiero (2 050 millones de dólares), situación que llevó a la mayor descapitalización del país que se tradujo en la hiperinflación más alta del Perú y Latinoamérica. El gobierno de Fujimori alcanza éste record de pago a nivel de los intereses con 7190 millones de dólares en su primer quinquenio. El cuadro excluye los 4 873 millones de dólares que ha refinanciado con Bonos Brady hechos efectivos en 1997 (segundo quinquenio de Fujimori), lo cual solo ha incrementado los intereses a pagar, en términos prácticos.

\section{CONCLUSIONES}

El informe presentado permite mostrar que, macro económicamente, el sistema de la deuda externa pública periodos políticos, quinquenales en su mayoría, para demostrar el comportamiento de la deuda asociado a los diferentes gobiernos.

El análisis de la deuda externa muestra que entre 1970 y el 2003 se ha recibido un total de 32656 millones de dólares y se ha pagado un total de 58062 millones de dólares, de los cuales 31513 son amortizaciones. A pesar de haber pagado un monto similar al desembolsado, aún se sigue debiendo cerca de 22000 millones de dólares y no los 1000 millones de diferencia.

Ello obedece a la dinámica del capital financiero y a las condiciones impues-

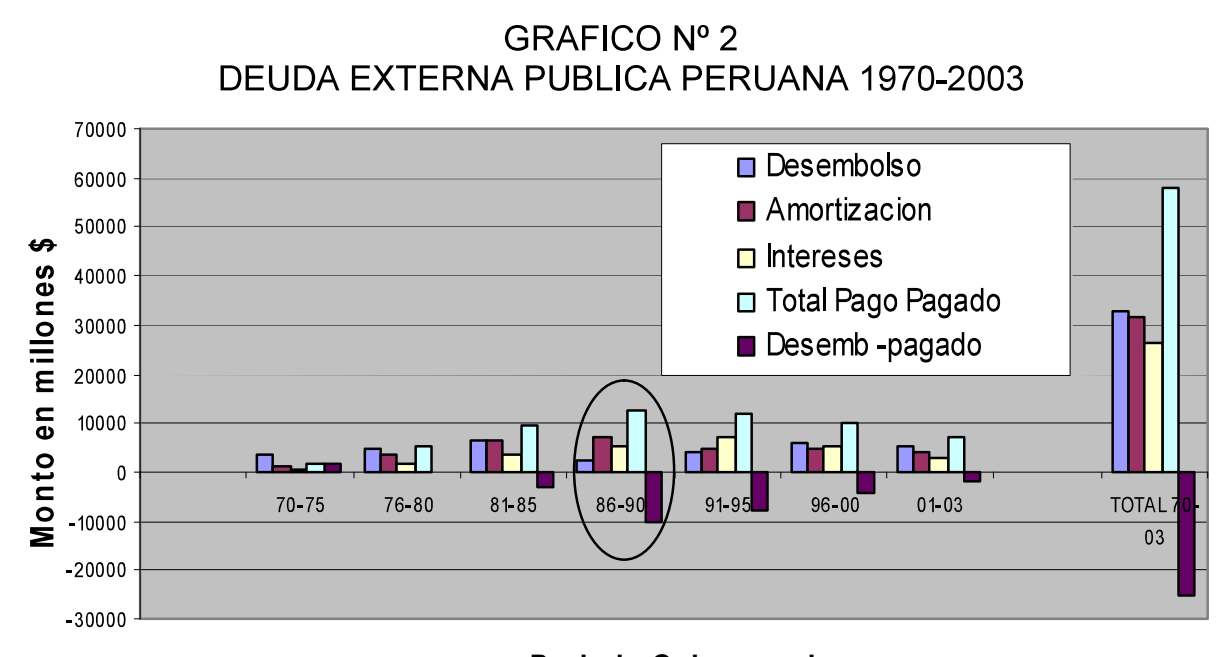

Periodo Quinquenal

Fuente: Compendio Estadistico 1993-94. Tomo 3. 245-248 y 289-296. INEI (1). BCR. Memorias 2000, 2002 y 2003 (5-7). 
es un mecanismo que impide reinvertir recursos generados internamente, para dedicarlos al pago del servicio de la deuda externa. Se puede apreciar que si sumamos amortización e intereses, se ha pagado casi el doble de lo prestado.

El pago neto promedio anual (Pago menos desembolsos) de los últimos
20 años ha significado una descapitalización del país.

Dejando de pagar 500 millones de dólares del servicio de la deuda externa se podría duplicar las remuneraciones a los maestros y dejando de pagar 1000 millones de dólares, podrían invertirse en el agro para revertir su situación actual.
Esto último evidentemente con una serie de políticas y apoyo técnicos adecuados a la realidad local.

\section{REFERENCIAS BIBLIOGRAFICAS}

1. Instituto Nacional de Estadística e Informática. Perú Compendio Estadístico 1993-1994. Tomo 3. pp 243, 245, 246, 249, 289, 290, 291-296. Lima: INEI; Julio 1994.

2. Instituto Nacional de Estadística e Informática. Perú Series Estadísticas 1970-94. pp. 319-329. Lima: INEl; Octubre 1995.

3. Instituto Nacional de Estadística e Informática. Compendio de Estadísticas Sociales 1995-1996. Lima: INEl; Octubre 1996.

4. Instituto Nacional de Estadística e Informática. Perú Compendio Estadístico 1996-1997. Lima: INEl; Julio 1997.

5. Banco Central de Reserva. Memoria Anual 2000. Lima: BCRP; marzo 2001.

6. Banco Central de Reserva. Memoria Anual 2002. Sección IV. pp. 100-110 y Cuadros Anexos. Lima: BCRP; marzo 2003.

7. Banco Central de Reserva. Memoria Anual 2003. Lima: BCRP; marzo 2004.

8. Banco Central de Reserva. Nota Semanal. Resumen Informativo № 28. Cuadros anexos. Lima: BCRP; 9 julio 2004

9. Yepez, Isabel y Bernedo Alvarado, J. La sindicalización en el Perú. Lima: Pontificia Universidad Católica del Perú;1981

10. Yepez, Isabel y Sulmont, Denis. Trabajo en cifras. Programa Académico de Ciencias sociales. Serie: Proyección Social, pp. 53, 56. Lima: Pontificia Universidad Católica del Perú; mayo 1983.

11. Cuanto S.A. Perú en Números. Lima: Cuanto; 1991

12. Cuanto S.A. Perú en Números. Lima: Cuanto; 1993

13. International Monetary Fund. External Debt Statistics. Guide for Compilers and Users. Washington: IMF; 2003.

14. Portocarrero, Felipe; Arlette, Beltrán; Romero, María Elena. Compendio Estadístico del Perú 1900-1990. Lima, Universidad del Pacífico, Centro de Investigaciones. 1992

Email: jorospi@terra.com.pe jorospi@ec-red.com 